\title{
A Continuous Model for the Dynamical Analysis of Mistuned Bladed Rotors
}

\author{
Paolo Bisegna ${ }^{1}$ and Giovanni Caruso ${ }^{2}$ \\ ${ }^{1}$ Department of Civil Engineering, University of Rome Tor Vergata, Via Politecnico 1, 00133 Rome, Italy \\ ${ }^{2}$ Construction Technologies Institute, National Research Council, Via Salaria Km 29,300, 00016 Monterotondo Stazione, Rome, Italy
}

Correspondence should be addressed to Giovanni Caruso, g.caruso@itc.cnr.it

Received 26 October 2011; Revised 23 January 2012; Accepted 12 February 2012

Academic Editor: Meinhard Taher Schobeiri

Copyright ( $\odot 2012$ P. Bisegna and G. Caruso. This is an open access article distributed under the Creative Commons Attribution License, which permits unrestricted use, distribution, and reproduction in any medium, provided the original work is properly cited.

Ideal bladed rotors are rotationally symmetric, as a consequence they exhibit couples of degenerate eigenmodes at coinciding frequencies. When even small imperfections are present destroying the periodicity of the structure (disorder or mistuning), each couple of degenerate eigenfrequencies splits into two distinct values (frequency split) and the corresponding modal shapes exhibit vibration amplitude peaks concentrated around few blades (localization phenomenon). In this paper a continuous model describing the in-plane vibrations of a mistuned bladed rotor is derived via the homogenization theory. Imperfections are accounted for as deviations of the mass and/or stiffness of some blades from the design value; a perturbation approach is adopted in order to investigate the frequency split and localization phenomena arising in the imperfect structure. Numerical simulations show the effectiveness of the proposed model, requiring much lower computational effort than classical finite element schemes.

\section{Introduction}

Bladed rotors used in turbomachinery possess rotational symmetry provided that no imperfections are present, that is, if all the blades and associated disk sectors are identical to each other. Under this ideal condition, their dynamical behavior is characterized by the presence of couples of degenerate eigenmodes at coinciding frequencies, as shown in $[1,2]$. That behavior can be studied in detail by using the finite element technique $[1,3,4]$, and the computational cost may be significatively reduced by exploiting the periodicity properties characterizing these structures. In fact, for a given structural eigenmode, all the blades exhibit the same vibration amplitude with a constant phase shift between adjacent ones; thus only a single sector of the structure may be considered in the analysis, by enforcing suitable constraints depending on the admissible phase shifts [1].

As a matter of fact, in practice the symmetry is destroyed by the presence of unavoidable manufacturing defects, material tolerances, or damage arising during service. The loss of symmetry, called disorder or mistuning, may significantly alter the system dynamics even at small disorder level. In particular, the eigenmode degeneracy is removed, and the coinciding modal frequencies of a degenerate eigenmode are split into two distinct values (frequency split phenomenon). Moreover, the vibration localization effect may appear [57], consisting of a vibrational-energy confinement in small regions of the rotor, rapidly decaying far away. As a consequence, some blades may vibrate with small amplitude whereas some others with significantly larger amplitude, causing a local stress increase, possibly leading to fatigue failure.

This phenomenon has been extensively studied in the literature: recent works studied the vibration localization arising in bladed rotors by employing the Galerkin method $[8,9]$, the finite element method [10], or by considering simplified lumped-parameter models [11-15]. Both lumpedparameter models and finite element analysis were employed in [16] to investigate the dependence of the localization phenomenon from the internal damping and interblade coupling in bladed disks. Finite element analysis applied to the study of disordered bladed rotors with a large number of blades is quite accurate but may require a significant 
amount of computational effort. On the other hand, simplified lumped-parameter models supply satisfying qualitative results, being also computationally more effective than finite element schemes, but may not be able to capture all the peculiarities of the dynamical behavior of a complex structure such as a bladed rotor.

Less effort has been devoted to the derivation of continuous models, which can realistically describe the dynamics of imperfect bladed rotors with low computational effort. For this reason they are particularly useful for performing parametric analyses or designing innovative vibration control schemes. Moreover, continuous models can easily yield analytical results, thus providing a general and comprehensive understanding of the phenomena involved. A continuous model of a bladed rotor without imperfections was considered in [17]. The rotor was composed of grouped blades mounted on a flexible disk. The coupling between adjacent groups of blades due to the disk was accounted for by using lumped springs, whereas distributed springs were used to model the blade stiffness. In [18] the homogenization technique was applied to a simple lumped-parameter model, composed of pendula acted upon by rotational and linear springs, widely employed in the literature to qualitatively study the localization phenomenon in bladed rotors. A continuous model was obtained, yielding analytical closed-form expressions for the eigenfrequencies and the eigenmodes, as well as for the resonance peaks of the forced response, depending on the mistuning level. In [19] a homogenized continuous model of a bladed rotor without imperfections was developed, taking into account the flexibility of both the support disk and the blades by means of the Euler-Bernoulli beam theory. That continuous model was used for the design and optimization of a passive vibration control scheme.

In this paper the model proposed in [19] is suitably generalized to account for the presence of mistuning. In particular, the in-plane vibrations of a mistuned bladed rotor are considered. In Section 2.1 a classical model of a bladed rotor is recalled, employing the Euler-Bernoulli beam theory for the description of both the blades and the support ring. The equations governing that model are solved using the finite element technique, and the obtained numerical results are used as a benchmark for the validation of the homogenized model proposed here. In Section 2.2 the homogenization technique is applied to the Euler-Bernoulli model in order to obtain a continuous homogenized model of the bladed rotor without imperfections. In Section 2.3 the homogenized model previously derived is generalized in order to account for the presence of imperfections, consisting of deviations of the mass and/or stiffness of some blades from the design value. In Section 3.1 analytical expressions of the natural frequencies and vibration modes of a rotor without imperfections are computed using the homogenized model. In order to derive analytical expressions of the same quantities relevant to a rotor with imperfections, a linearization of the proposed homogenized model is performed in Section 3.2, by employing a perturbation technique. As a result, a linear variational formulation of the homogenized model is obtained, supplying analytical expressions for the natural frequencies and vibration mode of a mistuned bladed rotor

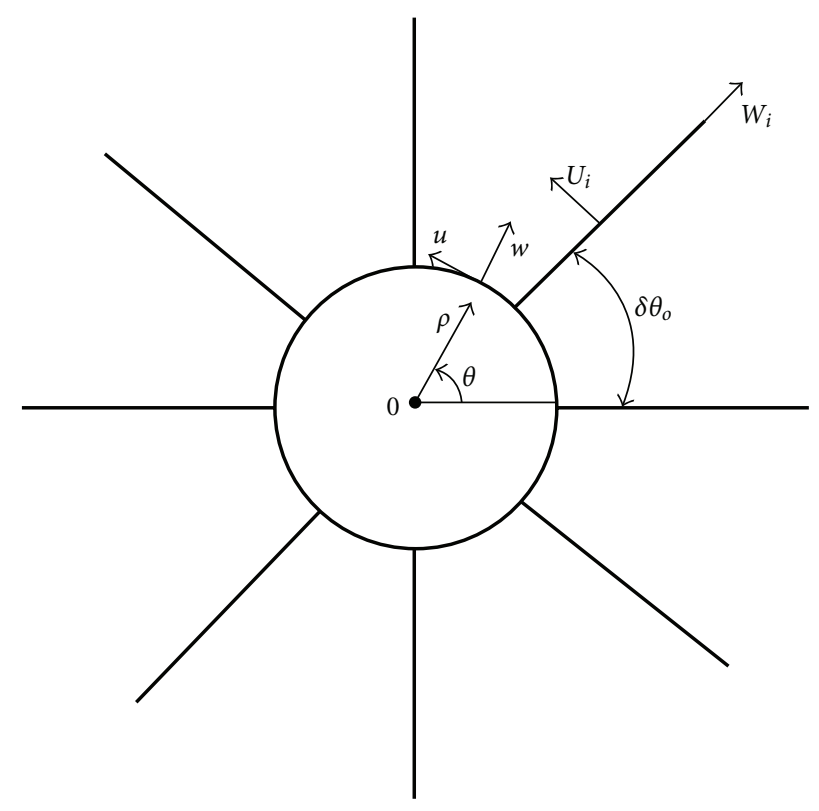

FIGURE 1: Schematic representation of a bladed rotor.

(Section 3.3). Finally, some simulation results are presented in Section 4, showing the ability of the proposed homogenized model to describe the dynamical behavior of the mistuned bladed rotor introduced in Section 4.1. In particular the results supplied by the homogenized model, obtained by using a numerical approach, and the analytical results supplied by the linearized version of the homogenized model are compared with results supplied by the classical Euler-Bernoulli model, obtained using the finite element technique as described in Section 4.2. The comparison shows good agreement between the finite-element and the homogenized model outcomes, relevant to both the frequency split and the localized vibration modes of the mistuned rotor (Sections 4.4 and 4.5).

\section{Homogenized Model}

In this section a homogenized model for the analysis of the in-plane vibrations of a mistuned bladed rotor is derived. The rotor is schematically represented in Figure 1; it is made of a linearly elastic material and is composed of $N_{b}$ blades, of length $l_{b}$, clamped on a ring of radius $R$, representing the turbine shaft. The angular spacing $\delta \theta_{o}$ between any two adjacent blades is constant and equal to $2 \pi / N_{b}$.

2.1. Euler-Bernoulli Model. In order to develop the homogenized model, a classical model based on the Euler-Bernoulli theory is briefly recalled here, denoted in the foregoing with the acronym EB. It will be also used as a benchmark, after discretization by means of the finite-element method, in order to assess the accuracy of the homogenized model in performing a dynamical analysis of a mistuned rotor. A polar coordinate system $(O, \rho, \theta)$ is introduced. Let $u(\theta)$ and $w(\theta)$ be the tangential and radial displacement of the ring, respectively; let $U_{i}(\rho)$ and $W_{i}(\rho)$ be the transversal and 
axial displacement of the $i$ th blade, placed at an angle $\theta_{i}$, respectively, as shown in Figure 1. Both the ring and the blades are assumed to be inextensible, and their flexural behavior is governed by the Euler-Bernoulli theory. The axial strain $\varepsilon$, the rotation $\varphi$, and the variation of curvature $\Delta \chi$ of the cross section of the ring are respectively given by

$$
\begin{gathered}
\varepsilon=\frac{1}{R}\left(\frac{\partial u}{\partial \theta}+w\right), \quad \varphi=\frac{1}{R}\left(u-\frac{\partial w}{\partial \theta}\right), \\
\Delta \chi=\frac{1}{R^{2}}\left(\frac{\partial u}{\partial \theta}-\frac{\partial^{2} w}{\partial \theta^{2}}\right),
\end{gathered}
$$

where $R$ is the ring radius.

Assuming first that no imperfections are present, and denoting with $\sigma_{r}$ and $K_{r}$, respectively, the linear mass density and in-plane bending stiffness of the ring, and with $\sigma_{o b}$ and $K_{o b}$, respectively, the linear mass density and bending stiffness of each blade, possibly depending on the radial coordinate $\rho$, the following Hamiltonian functional describing the dynamical behavior of the structure can be written as follows:

$$
\begin{aligned}
\mathscr{H}= & \frac{1}{2} \int_{0}^{2 \pi} \sigma_{r}\left[\left(\frac{\partial u}{\partial t}\right)^{2}+\left(\frac{\partial w}{\partial t}\right)^{2}\right] R d \theta \\
& +\frac{1}{2} \sum_{i=1}^{N_{b}} \int_{R}^{R+l_{b}} \sigma_{o b}(\rho)\left[\left(\frac{\partial U_{i}}{\partial t}\right)^{2}+\left(\frac{\partial W_{i}}{\partial t}\right)^{2}\right] d \rho \\
& -\frac{1}{2} \int_{0}^{2 \pi} \frac{K_{r}}{R^{4}}\left(\frac{\partial u}{\partial \theta}-\frac{\partial^{2} w}{\partial \theta^{2}}\right)^{2} R d \theta \\
& -\frac{1}{2} \sum_{i=1}^{N_{b}} \int_{R}^{R+l_{b}} K_{o b}(\rho)\left(\frac{\partial^{2} U_{i}}{\partial \rho^{2}}\right)^{2} d \rho,
\end{aligned}
$$

subjected to the following constraints:

$$
\begin{gathered}
\frac{1}{R}\left(\frac{\partial u}{\partial \theta}+w\right)=0, \quad \theta \in(0,2 \pi) ; \\
\frac{\partial W_{i}}{\partial \rho}=0, \quad \rho \in\left(R, R+l_{b}\right), i=1 \cdots N_{b} ; \\
W_{i}=w, \quad U_{i}=u, \\
\frac{\partial U_{i}}{\partial \rho}=\frac{1}{R}\left(u-\frac{\partial w}{\partial \theta}\right), \quad \rho=R, \theta=\theta_{i}, i=1 \cdots N_{b},
\end{gathered}
$$

where $t$ denotes the time.

Many typologies of imperfections can be accounted for by varying the linear mass density and/or bending stiffness of some blades with respect to the design value; for example, in [15] a crack in a single blade was modeled by reducing the bending stiffness of the damaged blade. Accordingly, if the $j$ th blade of the rotor is imperfect, the linear mass density $\sigma_{b j}(\rho)$ and bending stiffness $K_{b j}(\rho)$ of that blade can be modified as follows:

$$
\begin{aligned}
\sigma_{b j}(\rho) & =\sigma_{o b}(\rho)+\delta \sigma_{b j}(\rho), \\
K_{b j}(\rho) & =K_{o b}(\rho)+\delta K_{b j}(\rho),
\end{aligned}
$$

where $\delta \sigma_{b j}$ and $\delta K_{b j}$ are the variations with respect to the corresponding nominal values.
2.2. Homogenized Model for a Perfect Rotor. The homogenized model developed in [19], valid for a rotor without imperfections, is briefly recalled here in order to illustrate the homogenization procedure. The variational formulation (2) is rewritten for a family of rotors with increasing number $N$ of blades, starting from $N=N_{b}$, which is the number of blades exhibited by the reference rotor, in order to approach the homogenization limit $N \rightarrow \infty$. It is assumed that, for each chosen $N$, the blade mass density $\sigma_{o b}^{N}$ and bending stiffness $K_{o b}^{N}$ rescale as $\sigma_{o b}(\rho)\left(N_{b} / N\right)$ and $K_{o b}(\rho)\left(N_{b} / N\right)$, respectively, such that the overlined quantities,

$$
\bar{\sigma}_{o b}(\rho)=\frac{\sigma_{o b}^{N}}{\delta \theta}, \quad \bar{K}_{o b}(\rho)=\frac{K_{o b}^{N}}{\delta \theta},
$$

where $\delta \theta=2 \pi / N$ is the angular spacing between adjacent blades, remain constant during the homogenization limit. The quantities defined in (5) represent, respectively, the homogenized linear mass density and bending stiffness. These assumptions are substituted into the functional (2) and the homogenization limit $N \rightarrow \infty$ is then performed; by extending the functions $U_{i}(\rho)$ and $W_{i}(\rho), i=1 \cdots N$ to the annular region $\left(R, R+l_{b}\right) \times(0,2 \pi)$ and assuming that they converge, together with their derivatives, to functions $U(\rho, \theta)$ and $W(\rho, \theta)$, the functional (2) converges, for $N \rightarrow \infty$, to [19]

$$
\begin{aligned}
\mathcal{H}_{\mathrm{hom}}= & \frac{1}{2} \int_{0}^{2 \pi} \sigma_{r}\left[\left(\frac{\partial U}{\partial t}\right)^{2}+\left(-\frac{\partial^{2} U}{\partial \theta \partial t}\right)^{2}\right]_{\rho=R} R d \theta \\
& +\frac{1}{2} \int_{0}^{2 \pi} \int_{R}^{R+l_{b}} \bar{\sigma}_{o b}(\rho)\left(\frac{\partial U}{\partial t}\right)^{2} d \rho d \theta \\
& +\frac{1}{2} \int_{0}^{2 \pi}\left(\int_{R}^{R+l_{b}} \bar{\sigma}_{o b}(\rho) d \rho\right)\left[-\frac{\partial^{2} U}{\partial \theta \partial t}\right]_{\rho=R}^{2} d \theta \\
& -\frac{1}{2} \int_{0}^{2 \pi} \frac{K_{r}}{R^{4}}\left[\frac{\partial U}{\partial \theta}+\frac{\partial^{3} U}{\partial \theta^{3}}\right]_{\rho=R}^{2} R d \theta \\
& -\frac{1}{2} \int_{0}^{2 \pi} \int_{R}^{R+l_{b}} \bar{K}_{o b}(\rho)\left(\frac{\partial^{2} U}{\partial \rho^{2}}\right)^{2} d \rho d \theta,
\end{aligned}
$$

subjected to the following constraints:

$$
\frac{\partial U}{\partial \rho}=\frac{1}{R}\left(U+\frac{\partial^{2} U}{\partial \theta^{2}}\right), \quad \rho=R, \theta \in(0,2 \pi)
$$

In order to analyze the rotor dynamics, a sinusoidal time dependence of the unknown $U$ is assumed, that is,

$$
U(\rho, \theta, t)=V(\rho, \theta) \mathrm{e}^{i \omega t},
$$




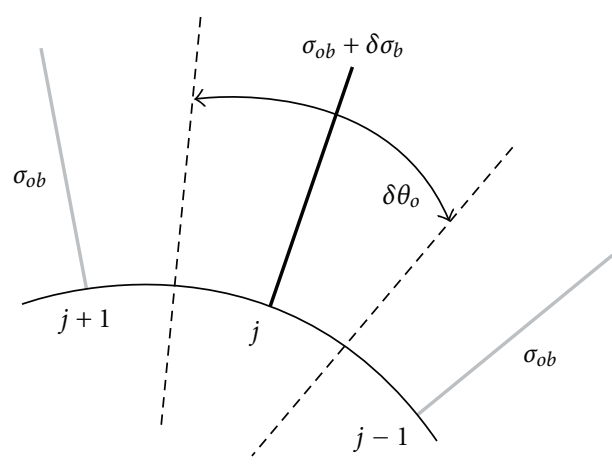

(a)

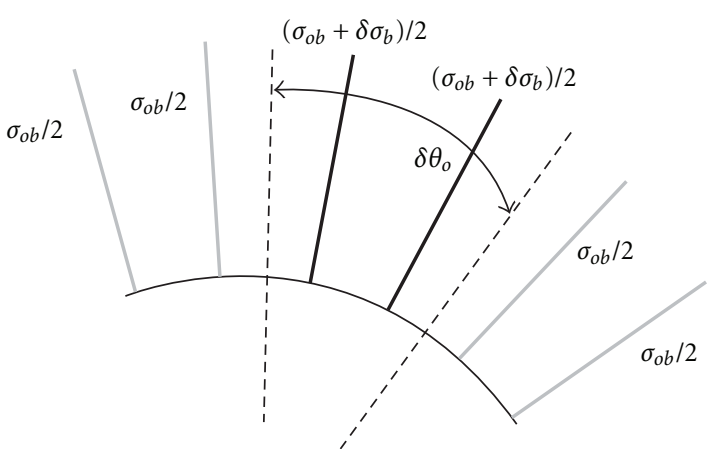

(b)

FIgURE 2: Homogenization limit for an imperfect rotor; imperfection due to an increase of linear mass density of a blade. (a) Reference rotor with $N_{b}$ blades and imperfection on the $j$ th blade, in black color. (b) Homogenization step process $k=1$; rotor with $N=2 N_{b}$ blades, with rescaled bending stiffness and linear mass density.

where $i=\sqrt{-1}$ is the imaginary unit. Enforcing this position and recasting the functional (6) into a variational form, the following is obtained:

$$
\begin{aligned}
& -\omega^{2}\left\{\int_{0}^{2 \pi} \sigma_{r}\left[V \psi+\frac{\partial V}{\partial \theta} \frac{\partial \psi}{\partial \theta}\right]_{\rho=R} R d \theta\right. \\
& +\int_{0}^{2 \pi} \int_{R}^{R+l_{b}} \bar{\sigma}_{o b}(\rho) V \psi d \rho d \theta \\
& \left.\quad+\int_{0}^{2 \pi}\left(\int_{R}^{R+l_{b}} \bar{\sigma}_{o b}(\rho) d \rho\right)\left[\frac{\partial V}{\partial \theta} \frac{\partial \psi}{\partial \theta}\right]_{\rho=R} d \theta\right\} \\
& +\int_{0}^{2 \pi} \frac{K_{r}}{R^{4}}\left[\left(\frac{\partial V}{\partial \theta}+\frac{\partial^{3} V}{\partial \theta^{3}}\right)\left(\frac{\partial \psi}{\partial \theta}+\frac{\partial^{3} \psi}{\partial \theta^{3}}\right)\right]_{\rho=R} R d \theta \\
& +\int_{0}^{2 \pi} \int_{R}^{R+l_{b}} \bar{K}_{o b}(\rho) \frac{\partial^{2} V}{\partial \rho^{2}} \frac{\partial^{2} \psi}{\partial \rho^{2}} d \rho d \theta=0,
\end{aligned}
$$

subjected to the following constraints:

$$
\begin{gathered}
\frac{\partial V}{\partial \rho}=\frac{1}{R}\left(V+\frac{\partial^{2} V}{\partial \theta^{2}}\right), \\
\frac{\partial \psi}{\partial \rho}=\frac{1}{R}\left(\psi+\frac{\partial^{2} \psi}{\partial \theta^{2}}\right), \quad \rho=R, \theta \in(0,2 \pi),
\end{gathered}
$$

where $\psi(\rho, \theta)$ indicates a test function. The homogenized model described here is denoted with the acronym HOM in the foregoing.

2.3. Homogenized Model for an Imperfect Rotor. The presence of imperfections can be accounted for in the homogenized model by letting the homogenized linear mass density and bending stiffness depend also on the angular variable $\theta$. Accordingly, it is assumed that

$$
\begin{aligned}
\bar{\sigma}_{b}(\rho, \theta) & =\bar{\sigma}_{o b}(\rho)+\delta \bar{\sigma}_{b}(\rho, \theta), \\
\bar{K}_{b}(\rho, \theta) & =\bar{K}_{o b}(\rho)+\delta \bar{K}_{b}(\rho, \theta),
\end{aligned}
$$

where $\delta \bar{\sigma}_{b}$ and $\delta \bar{K}_{b}$ are suitable perturbations superimposed to the homogenized design values of linear mass density $\bar{\sigma}_{o b}$ and bending stiffness $\bar{K}_{o b}$, respectively. In order to choose $\delta \bar{\sigma}_{b}$ and $\delta \bar{K}_{b}$, it is necessary to specify how the imperfect blades behave during the homogenization limit. For the sake of example, it is assumed that the $j$ th blade of the rotor is imperfect, with increased linear mass density equal to $\sigma_{o b}+\delta \sigma_{b j}$ as indicated in Figure 2. When the number of blades is increased to approach the homogenization limit, the number of imperfect blades increases as depicted in Figure 2, and their mass density and bending stiffness are rescaled according to the conditions given in Section 2.2. In particular, at the $k$ th homogenization-process step, the total number of blades becomes $N=2^{k} N_{b}$, and the blade linear mass density and bending stiffness are rescaled by a factor $1 / 2^{k}$ with respect to their nominal value; on the other hand, the number of imperfect blades becomes $2^{k}$, with rescaled linear mass density variation equal to $\delta \sigma_{b j} / 2^{k}$.

Under these assumptions, in the homogenization limit $N \rightarrow \infty$, the homogenized linear mass density perturbation $\delta \bar{\sigma}_{b}$ and the homogenized bending stiffness perturbation $\delta \bar{K}_{b}$ are piecewise constant functions having the following expressions:

$$
\begin{aligned}
\delta \bar{\sigma}_{b}(\rho, \theta) & =\frac{\delta \sigma_{b j}(\rho)}{\delta \theta_{o}} \chi_{j}(\theta), \\
\delta \bar{K}_{b}(\rho, \theta) & =\frac{\delta K_{b j}(\rho)}{\delta \theta_{o}} \chi_{j}(\theta),
\end{aligned}
$$

where

$$
\begin{gathered}
\chi_{j}(\theta)=1, \quad \theta \in\left(\theta_{j}-\frac{\delta \theta_{o}}{2}, \theta_{j}+\frac{\delta \theta_{o}}{2}\right), \\
\chi_{j}=0, \quad \text { elsewhere. }
\end{gathered}
$$

The expression (12) can be straightforwardly generalized to the case of multiple imperfect blades.

\section{Analytical Solutions for Eigenfrequencies and Eigenmodes}

In this section explicit formulas for the eigenfrequencies and eigenmodes of a bladed rotor, with or without imperfections, 
are reported. They are obtained by using the homogenized model developed in Sections 2.2 and 2.3.

3.1. Perfect Rotor. In order to evaluate the eigenmodes and eigenfrequencies of a perfect rotor by using the homogenized model proposed here, it is assumed for the sake of simplicity that $\bar{\sigma}_{o b}$ and $\bar{K}_{o b}$ do not depend on the radial variable $\rho$; under this simplifying assumption, using the localization lemma, (9) and (10) yields the field equilibrium equation:

$$
-\omega^{2} \bar{\sigma}_{o b} V+\bar{K}_{o b} \frac{\partial^{4} V}{\partial \rho^{4}}=0, \quad(\rho, \theta) \in\left(R, R+l_{b}\right) \times(0,2 \pi),
$$

and the boundary conditions:

$$
\begin{aligned}
& -\omega^{2} \sigma_{r} R\left(V-\frac{\partial^{2} V}{\partial \theta^{2}}\right)+\omega^{2} \bar{\sigma}_{o b} l_{b} \frac{\partial^{2} V}{\partial \theta^{2}} \\
& -\frac{K_{r}}{R^{3}}\left(\frac{\partial}{\partial \theta}+\frac{\partial^{3}}{\partial \theta^{3}}\right)^{2} V-\frac{\bar{K}_{o b}}{R}\left(1+\frac{\partial^{2}}{\partial \theta^{2}}\right) \frac{\partial^{2} V}{\partial \rho^{2}} \\
& -\bar{K}_{o b} \frac{\partial^{3} V}{\partial \rho^{3}}=0, \quad \rho=R, \theta \in(0,2 \pi) ; \\
& \frac{\partial V}{\partial \rho}=\frac{1}{R}\left(V+\frac{\partial^{2} V}{\partial \theta^{2}}\right), \quad \rho=R, \theta \in(0,2 \pi) ; \\
& \bar{K}_{o b} \frac{\partial^{2} V}{\partial \rho^{2}}=0, \quad \rho=R+l_{b}, \theta \in(0,2 \pi) ; \\
& -\bar{K}_{o b} \frac{\partial^{3} V}{\partial \rho^{3}}=0, \quad \rho=R+l_{b}, \theta \in(0,2 \pi) .
\end{aligned}
$$

Due to the rotational symmetry of the problem, the eigenmodes $V$ depend harmonically on the angular variable $\theta$. For each fixed nodal diameter, denoted by the modal number $n \geq 0$, a countable set of eigenfrequencies and eigenmodes, denoted by the modal number $l=1,2,3 \ldots$, can be computed as follows. Fixed $n$, a solution of the field equation (14), is given by

$$
V_{o n, l}(\rho, \theta)=2 A_{n, l}\left(\sum_{i=1}^{4} C_{i} f_{i}(\rho)\right) \cos \left(n \theta+\varphi_{n, l}\right),
$$

where $A_{n, l}$ is an arbitrary constant,

$$
\begin{aligned}
f_{1}(\rho)=\cos \left[\lambda_{n, l}(\rho-R)\right], & f_{2}(\rho)=\sin \left[\lambda_{n, l}(\rho-R)\right], \\
f_{3}(\rho)=\cosh \left[\lambda_{n, l}(\rho-R)\right], & f_{4}(\rho)=\sinh \left[\lambda_{n, l}(\rho-R)\right]
\end{aligned}
$$

and the modal eigenfrequency $\omega_{o n, l}$ is related to $\lambda_{n, l}$ by the relation

$$
\omega_{o n, l}=\sqrt{\frac{\bar{K}_{o b}}{\bar{\sigma}_{o b}}} \lambda_{n, l}^{2}
$$

In (16) the quantities $C_{i}, i=1 \cdots 4$, are scalar unknowns; by substituting (16) into the four boundary conditions (15), an eigenvalue problem is obtained, exhibiting a countable set of eigenvalues $\lambda_{n, l}$ and corresponding eigenvectors $C_{i}$, $i=1 \cdots 4$. The phase orientation $\varphi_{n, l}$ is left undetermined after solving (14) and (15); as a consequence, for each fixed $n>0$ and $l$ two independent eigenmodes exist, at the same modal frequency $\omega_{\text {on, },}$. These are known in the literature as degenerate eigenmodes $[1,2]$. All the eigenmodes relevant to $n=0$ are nondegenerate.

\subsection{Linearized Homogenized Model for an Imperfect Rotor.} In order to derive analytical expressions for the modal eigenfrequencies and eigenmodes of the rotor in the presence of imperfections, the homogenized model proposed in Section 2.3 is linearized by employing a perturbation technique. Accordingly, the eigenmodes of the imperfect rotor are computed by perturbing the eigenmodes relevant to the perfect rotor, that is, for each fixed couple of modal indices $(n, l)$, the eigenmodes $V_{n, l}$ of the imperfect rotor are given by

$$
V_{n, l}(\rho, \theta)=V_{o n, l}(\rho, \theta)+\delta V_{n, l}(\rho, \theta),
$$

where $V_{o n, l}$ is the eigenmode of the perfect rotor given in (16) and $\delta V_{n, l}$ is an unknown perturbation. Moreover the relevant eigenfrequency $\omega_{n, l}$ is given by

$$
\omega_{n, l}=\omega_{o n, l}+\delta \omega_{n, l},
$$

where $\delta \omega_{n, l}$ is the frequency shift due to the presence of imperfections. By substituting the expansions (11), (19), and (20) into the weak formulation (9) and retaining only the first order terms, the following linearized weak formulation is obtained:

$$
\begin{aligned}
& -\omega_{o n, l}^{2}\left\{\left.\int_{0}^{2 \pi} \sigma_{r}\left(\delta V_{n, l} \psi+\frac{\partial \delta V_{n, l}}{\partial \theta} \frac{\partial \psi}{\partial \theta}\right)\right|_{\rho=R} R d \theta\right. \\
& +\int_{0}^{2 \pi} \int_{R}^{R+l_{b}} \bar{\sigma}_{b} \delta V_{n, l} \psi d \rho d \theta \\
& \left.+\left.\int_{0}^{2 \pi} \bar{\sigma}_{b} l_{b} \frac{\partial \delta V_{n, l}}{\partial \theta} \frac{\partial \psi}{\partial \theta}\right|_{\rho=R} d \theta\right\} \\
& +\left.\int_{0}^{2 \pi} \frac{K_{r}}{R^{4}}\left(\frac{\partial \delta V_{n, l}}{\partial \theta}+\frac{\partial^{3} \delta V_{n, l}}{\partial \theta^{3}}\right)\left(\frac{\partial \psi}{\partial \theta}+\frac{\partial^{3} \psi}{\partial \theta^{3}}\right)\right|_{\rho=R} R d \theta \\
& +\int_{0}^{2 \pi} \int_{R}^{R+l_{b}} \bar{K}_{b} \frac{\partial^{2} \delta V_{n, l}}{\partial \rho^{2}} \frac{\partial^{2} \psi}{\partial \rho^{2}} d \rho d \theta \\
& =2 \omega_{o n, l} \delta \omega_{n, l}\left\{\left.\int_{0}^{2 \pi} \sigma_{r}\left(V_{o n, l} \psi+\frac{\partial V_{o n, l}}{\partial \theta} \frac{\partial \psi}{\partial \theta}\right)\right|_{\rho=R} R d \theta\right. \\
& +\int_{0}^{2 \pi} \int_{R}^{R+l_{b}} \bar{\sigma}_{b} V_{o n, l} \psi d \rho d \theta \\
& \left.+\left.\int_{0}^{2 \pi} \bar{\sigma}_{b} l_{b} \frac{\partial V_{o n, l}}{\partial \theta} \frac{\partial \psi}{\partial \theta}\right|_{\rho=R} d \theta\right\} \\
& +\omega_{o n, l}^{2}\left\{\int_{0}^{2 \pi} \int_{R}^{R+l_{b}} \delta \bar{\sigma}_{b} V_{o n, l} \psi d \rho d \theta\right. \\
& \left.+\int_{0}^{2 \pi} l_{b} \delta \bar{\sigma}_{b} \frac{\partial V_{o n, l}}{\partial \theta} \frac{\partial \psi}{\partial \theta}\right\} \\
& -\int_{0}^{2 \pi} \int_{R}^{R+l_{b}} \delta \bar{K}_{b} \frac{\partial^{2} V_{o n, l}}{\partial \rho^{2}} \frac{\partial^{2} \psi}{\partial \rho^{2}} d \rho d \theta,
\end{aligned}
$$


under the following constraints:

$$
\begin{gathered}
\frac{\partial \delta V_{n, l}}{\partial \rho}=\frac{1}{R}\left(\delta V_{n, l}+\frac{\partial^{2} \delta V_{n, l}}{\partial \theta^{2}}\right) \\
\frac{\partial \psi}{\partial \rho}=\frac{1}{R}\left(\psi+\frac{\partial^{2} \psi}{\partial \theta^{2}}\right), \quad \rho=R, \theta \in(0,2 \pi),
\end{gathered}
$$

where it is assumed, for simplicity, that $\bar{\sigma}_{o b}$ and $\bar{K}_{o b}$ do not depend on the radial variable $\rho$. The unknowns appearing in (21) are the scalar quantities $\delta \omega_{n, l}$ and $\varphi_{n, l}$ and the function $\delta V_{n, l}$. Due to the presence of the imperfections, the indeterminacy on the phase angle $\varphi_{n, l}$ is removed and, for each couple of modal indexes $(n, l)$, two set of solutions $\left(\delta \omega_{n, l}, \varphi_{n, l}, \delta V_{n, l}\right)$ are expected, corresponding to the two split eigenmodes belonging to the same degenerate eigenmode $V_{\text {on,l }}$ relevant to the perfect structure. This linearized homogenized model is denoted in the foregoing with the acronym L-HOM.

3.3. Eigenfrequencies and Eigenmodes Evaluation for an Imperfect Rotor. By considering the linearized variational formulation (21), it is observed that, for each fixed modal number $n$,

$$
\begin{aligned}
& g_{1}(\rho, \theta)=\left(\sum_{i=1}^{4} C_{i} f_{i}(\rho)\right) \cos (n \theta), \\
& g_{2}(\rho, \theta)=\left(\sum_{i=1}^{4} C_{i} f_{i}(\rho)\right) \sin (n \theta),
\end{aligned}
$$

with $C_{i}, i=1 \cdots 4$, and $\lambda_{n, l}$ solutions of (14) and (15), are two independent functions belonging to the kernel of the selfadjoint operator at the left hand side of (21). Accordingly, by substituting first $\psi=g_{1}$ and then $\psi=g_{2}$ in (21), together with the expression of $V_{o n, l}$ and $\omega_{o n, l}$ given by (16) and (18), respectively, two independent equations in the unknowns $\delta \omega_{n, l}$ and $\varphi_{n, l}$ are obtained, whose solutions supply the two couples of frequency split and phase orientation $\left(\delta \omega_{n, l}, \varphi_{n, l}\right)$ relevant to the perturbed mode $(n, l)$. Once the values of $\delta \omega_{n, l}$ and $\varphi_{n, l}$ have been computed, the corresponding modal shapes can be obtained by making use of the linear weak formulation (21). To this end (21) is integrated by parts with respect to the variable $\rho$ and, making use of $(22)_{2}$, it yields

$$
\begin{aligned}
& \int_{0}^{2 \pi} \int_{R}^{R+l_{b}}\left(-\omega_{o n, l}^{2} \bar{\sigma}_{b} \delta V_{n, l}+\bar{K}_{b} \frac{\partial^{4} \delta V_{n, l}}{\partial \rho^{4}}\right. \\
& -2 \omega_{o n, l} \delta \omega_{n, l} \bar{\sigma}_{b} V_{o n, l} \\
& \left.-\omega_{o n, l}^{2} \delta \bar{\sigma}_{b} V_{o n, l}+\delta \bar{K}_{b} \frac{\partial^{4} V_{n, l}}{\partial \rho^{4}}\right) \psi d \rho d \theta \\
& +\int_{0}^{2 \pi}\left\{-\omega_{o n, l}^{2} \sigma_{r} R\left(\delta V_{n, l} \psi+\frac{\partial \delta V_{n, l}}{\partial \theta} \frac{\partial \psi}{\partial \theta}\right)\right. \\
& -\omega_{o n, l}^{2} \bar{\sigma}_{b} l_{b} \frac{\partial \delta V_{n, l}}{\partial \theta} \frac{\partial \psi}{\partial \theta} \\
& +\frac{K_{r}}{R^{3}}\left(\frac{\partial \delta V_{n, l}}{\partial \theta}+\frac{\partial^{3} \delta V_{n, l}}{\partial \theta^{3}}\right)\left(\frac{\partial \psi}{\partial \theta}+\frac{\partial^{3} \psi}{\partial \theta^{3}}\right)
\end{aligned}
$$

$$
\begin{gathered}
-\frac{\bar{K}_{b}}{R} \frac{\partial \delta V_{n, l}}{\partial \rho^{2}}\left(\psi+\frac{\partial^{2} \psi}{\partial \theta^{2}}\right)+\bar{K}_{b} \frac{\partial^{3} \delta V_{n, l}}{\partial \rho^{3}} \psi \\
-2 \omega_{o n, l} \delta \omega_{n, l}\left[\sigma_{r} R\left(V_{o n, l} \psi+\frac{\partial V_{o n, l}}{\partial \theta} \frac{\partial \psi}{\partial \theta}\right)\right. \\
\left.+\bar{\sigma}_{b} l_{b} \frac{\partial V_{o n, l}}{\partial \theta} \frac{\partial \psi}{\partial \theta}\right] \\
-\omega_{o n, l}^{2} l_{b} \delta \bar{\sigma}_{b} \frac{\partial V_{o n, l}}{\partial \theta} \frac{\partial \psi}{\partial \theta} \\
+\delta \bar{K}_{b} \frac{\partial^{2} V_{o n, l}}{\partial \rho^{2}}\left(\psi+\frac{\partial^{2} \psi}{\partial \theta^{2}}\right) \\
+\left.\bar{K}_{b} \frac{\partial^{3} V_{o n, l}}{\partial \rho^{3}} \psi\right|_{\rho=R} ^{2 \pi}\left[\left(\bar{K}_{b} \frac{\partial^{2} \delta V_{n, l}}{\partial \rho^{2}}+\delta \bar{K}_{b} \frac{\partial^{2} V_{o n, l}}{\partial \rho^{2}}\right) \frac{\partial \psi}{\partial \rho}\right]_{\rho=R+l_{b}} d \theta \\
+\int_{0}^{2 \pi}\left[\left(-\bar{K}_{b} \frac{\partial^{3} \delta V_{n, l}}{\partial \rho^{3}}-\delta \bar{K}_{b} \frac{\partial^{3} V_{o n, l}}{\partial \rho^{3}}\right) \psi\right]_{\rho=R+l_{b}} d \theta=0 .
\end{gathered}
$$

Another weak-form equation is obtained by multiplying $(22)_{1}$ by $\psi$ and integrating over $(0,2 \pi)$, yielding

$$
\int_{0}^{2 \pi}\left[\frac{\partial \delta V_{n, l}}{\partial \rho}-\frac{1}{R}\left(\delta V_{n, l}+\frac{\partial^{2} \delta V_{n, l}}{\partial \theta^{2}}\right)\right] \psi d \theta=0 .
$$

In order to find out the unknown modal perturbation $\delta V_{n, l}$, a spectral representation is used by setting

$$
\begin{aligned}
& \delta V_{n, l}(\rho, \theta)=\sum_{i=1}^{4}\left\{\frac{C_{i 0}}{2}+\rho \frac{G_{i 0}}{2}\right. \\
&+\sum_{k>0}\left[\left(C_{i k}+\rho G_{i k}\right) \cos (k \theta)\right. \\
&\left.\left.\quad+\left(D_{i k}+\rho H_{i k}\right) \sin (k \theta)\right]\right\} f_{i}(\rho) .
\end{aligned}
$$

The representation (26) of $\delta V_{n, l}$ is then substituted in (24), and the test function $\psi$ is chosen according to the following expressions

$$
\begin{gathered}
\psi(\rho, \theta)=\cos (k \theta) \beta(\rho), \\
\psi(\rho, \theta)=\sin (k \theta) \beta(\rho),
\end{gathered}
$$

where $k$ is any positive fixed integer and $\beta$ is an arbitrary function of $\rho$. Using the localization lemma in the first integral of (24), an explicit expression for $G_{i k}$ and $H_{i k}, i=1 \cdots 4$, is obtained. The expression (26) for $\delta V_{n, l}$, where now $G_{i k}$ and $H_{i k}$ are known scalar quantities, is then substituted into (24) and (25). Linear equations in the unknown coefficients $C_{i k}$ and $D_{i k}$ are obtained, for each fixed positive integer $k$, by choosing the test function $\psi$ and $\partial \psi / \partial \rho$, at $\rho=R$ and $\rho=R+l_{b}$, as $\cos (k \theta)$ and $\sin (k \theta)$ and using again localization lemma. Their solution provides the Fourier coefficients of the unknown modal perturbation $\delta V_{n, l}$. 
TABLE 1: Modal frequencies $\omega_{\text {on,l }}$ in rad/s, relevant to the perfect rotor and evaluated according to the EB model. Modal frequencies relevant to modal numbers $n=2 \cdots 5$ and $l=1$.

\begin{tabular}{|c|c|c|c|c|c|c|c|}
\hline \multicolumn{2}{|c|}{$n=2$} & \multicolumn{2}{|c|}{$n=3$} & \multicolumn{2}{|c|}{$n=4$} & \multicolumn{2}{|c|}{$n=5$} \\
\hline 27.0746 & 27.0746 & 27.9718 & 27.9718 & 28.4242 & 28.4242 & 28.6596 & 28.6596 \\
\hline
\end{tabular}

\section{Numerical Simulations}

In this section, some numerical simulation results are presented in order to show the effectiveness of the proposed homogenized model in the investigation of the frequency split and localization phenomenon in imperfect rotors.

4.1. Case Study Bladed Rotor. A case study problem is introduced here; a bladed rotor, similar to the one schematically represented in Figure 1, is considered. It is composed of 32 elastic blades of cross-section $2 \times 50 \mathrm{~mm}$ and length $600 \mathrm{~mm}$; the blades are clamped to a support ring of radius $200 \mathrm{~mm}$ and cross section $4 \times 50 \mathrm{~mm}$. The ring and the blades are composed of steel, with Young modulus $210 \mathrm{GPa}$ and mass density $7850 \mathrm{Kg} / \mathrm{m}^{3}$. The degenerate modal frequencies of this bladed rotor are reported in Table 1, relevant to modal numbers $n=2 \cdots 5$ and $l=1$, and evaluated according to the EB model. The eigenmodes relevant to $n=0,1$ are not considered since they do not involve any deformation in the support ring, in fact for that modes $\Delta \chi=$ 0 [19].

4.2. Numerical Algorithms. In order to find a stationary solution for the Hamiltonian functional (2), describing the dynamical behavior of the case study bladed rotor according to the EB model, a finite-element scheme is adopted here. Accordingly, (2) is discretized by using two-node beam elements and Hermite polynomials as interpolation scheme, both for the ring and the blades. The axial inextensibility constraint is enforced by using a penalization method. A discrete formulation for the dynamical problem is finally obtained by assuming as unknowns the nodal displacements and rotations. The results provided by the latter discretized model are used in the foregoing as benchmark to assess the accuracy of the homogenized model.

The homogenized weak formulation in (9), defined in the annular region $\left(R, R+l_{b}\right) \times(0,2 \pi)$, is also discretized in the angular direction with the Ritz-Rayleigh method assuming, as shape functions,

$$
1, \quad 2 \cos (k \theta), \quad-2 \sin (k \theta), \quad k=1 \cdots n_{f},
$$

whereas a finite element discretization is assumed in the radial direction, employing two-node beam elements with Hermite polynomials as interpolation scheme. Accordingly, the nodal unknowns are the real and imaginary part of the complex Fourier coefficients of the tangential displacement $V$ and its radial derivative.

4.3. Validation of the Homogenization Limit. In this section, the homogenization limit is numerically studied, and a convergence analysis is presented. The modal eigenfrequencies of the perfect rotor described in Section 4.1 are computed

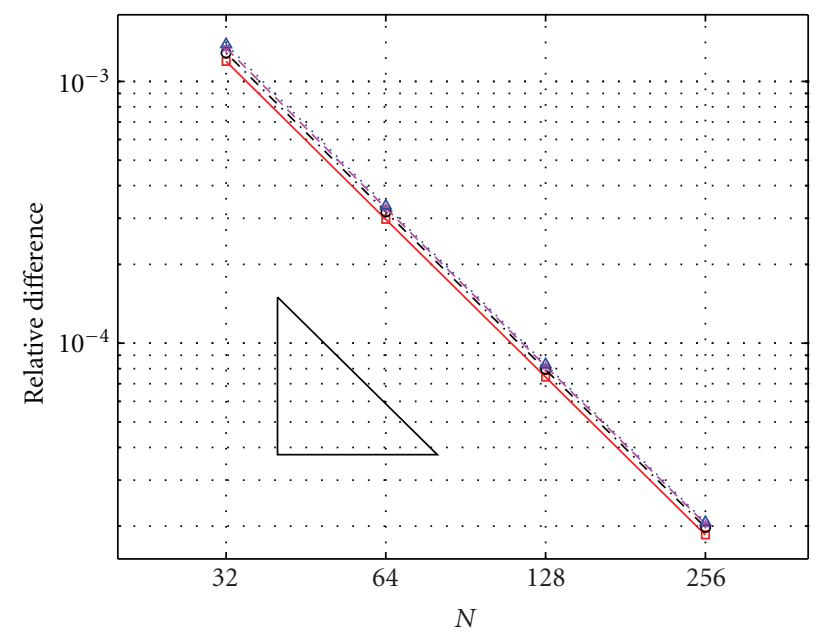

FIgure 3: Convergence analysis for the homogenization limit: relative error between modal frequencies evaluated according to the EB model and the HOM model, as a function of the number $N$ of blades. Rotor without imperfections. Modal frequencies relevant to modal numbers $l=1$ and $-\square n=2$; $-\cdot \circ n=3$; $--* n=4$; $\cdots \Delta n=5$. The triangle in the figure indicates the quadratic convergence slope.

increasing the number $N$ of blades, starting from the reference configuration with 32 blades and rescaling at the same time the linear mass density and the bending stiffness of the blades as described in Section 2, in order to approach the homogenization limit $N \rightarrow \infty$ relevant to the HOM model. In particular, $N=32, N=64, N=128$, and $N=256$ have been considered in the computations. In Figure 3 the relative error between degenerate eigenfrequencies evaluated according to the reference EB model and the HOM model is reported, relevant to modal numbers $n=2 \cdots 5$ and $l=1$. The figure shows that a quadratic convergence, indicated by the triangle, is achieved. The relative error slightly increases with the increase of $n$. The homogenized model turns out to be quite accurate in describing the rotor dynamics, as the relative error is very small even in the reference case of 32 blades.

4.4. Modal Frequency Analysis. In this section the modal frequencies relevant to an imperfect bladed rotor are analyzed. The imperfection is introduced by increasing the linear mass density of the fourth blade of the perfect rotor described in Section 4.1 by a factor $\gamma$. The modal frequencies $\omega_{n, l}$, relevant to modal numbers $n=2 \cdots 5$ and $l=1$, are evaluated according to the reference EB model and the HOM model, considering different values of $\gamma$. In Table 2 the modal frequencies evaluated according to the EB model are reported.

From Table 2 it can be seen that, due to the presence of imperfections, a frequency split occurs between frequencies belonging to the same modal number $(n, l)$, which were 
TABle 2: Modal frequencies $\omega_{n, l}$ in $\mathrm{rad} / \mathrm{s}$, relevant to the imperfect rotor and evaluated according to the EB model. The imperfection is introduced by increasing the mass of the fourth blade of the perfect rotor described in Section 4.1 by $\gamma=0.05 \%, 0.1 \%, 0.5 \%$, and $1 \%$, with respect to its nominal value. Modal frequencies relevant to modal numbers $n=2 \cdots 5$ and $l=1$.

\begin{tabular}{|c|c|c|c|c|c|c|c|c|}
\hline \multirow[b]{2}{*}{$\gamma=0.05 \%$} & \multicolumn{2}{|c|}{$n=2$} & \multicolumn{2}{|c|}{$n=3$} & \multicolumn{2}{|c|}{$n=4$} & \multicolumn{2}{|c|}{$n=5$} \\
\hline & 27.0742 & 27.0746 & 27.9713 & 27.9718 & 28.4237 & 28.4242 & 28.6591 & 28.6596 \\
\hline$\gamma=0.1 \%$ & 27.0738 & 27.0746 & 27.9709 & 27.9718 & 28.4233 & 28.4242 & 28.6587 & 28.6596 \\
\hline$\gamma=0.5 \%$ & 27.0703 & 27.0746 & 27.9671 & 27.9718 & 28.4193 & 28.4242 & 28.6544 & 28.6596 \\
\hline$\gamma=1 \%$ & 27.0656 & 27.0746 & 27.9619 & 27.9718 & 28.4134 & 28.4242 & 28.6476 & 28.6596 \\
\hline
\end{tabular}

TABLE 3: Relative error between frequency shifts relevant to the imperfect rotor, evaluated according to the HOM model and the EB model. The imperfection is introduced by increasing the mass of the fourth blade of the perfect rotor described in Section 4.1 by $\gamma=0.05 \%, 0.1 \%$, $0.5 \%$, and $1 \%$, with respect to its nominal value. Modal frequencies relevant to modal numbers $n=2 \cdots 5$ and $l=1$.

\begin{tabular}{lllll}
\hline & $n=2$ & $n=3$ & $n=4$ & $n=5$ \\
\hline$\gamma=0.05 \%$ & 0.0114 & 0.0281 & 0.0513 & 0.0824 \\
$\gamma=0.1 \%$ & 0.0116 & 0.0283 & 0.0519 & 0.0834 \\
$\gamma=0.5 \%$ & 0.0125 & 0.0299 & 0.0556 & 0.0906 \\
$y=1 \%$ & 0.0103 & 0.0261 & 0.0500 & 0.0828 \\
\hline
\end{tabular}

coinciding in the perfect case as shown in Table 1. The difference between eigenfrequencies relevant to the imperfect rotor in Table 2 and the corresponding ones relevant to the perfect rotor in Table 1 is the frequency shift $\delta \omega_{n, l}$, which occurs due to the presence of imperfections. It can be noticed that, for each couple of modal numbers $(n, l)$, one of the two frequency shifts $\delta \omega_{n, l}$ is vanishing; in fact, for each fixed $(n, l)$, one of the two eigenfrequencies of the imperfect rotor in Table 2 coincides with the corresponding frequency of the perfect rotor in Table 1. In particular, this eigenfrequency corresponds to a modal shape antisymmetric with respect to the location of the imperfect blade [18]. Table 3 contains the relative error between the frequency shifts $\delta \omega_{n, l}$ evaluated according to the EB model and to the HOM model. For each couple of modal numbers $(n, l)$, only the value relevant to the nonvanishing frequency shift is reported. The errors are almost independent from the imperfection level $\gamma$, and they slightly increase with the increase of $n$, ranging from $1 \%$ when $n=2$ to $9 \%$ when $n=5$. Finally, in Table 4 the relative errors between frequency shifts $\delta \omega_{n, l}$ evaluated according to the HOM model and the L-HOM model are reported. It is remarked here that while the results supplied by the HOM model are computed by using the numerical procedure described in Section 4.2, the results relevant to the L-HOM model are readily obtained using the analytical expressions developed in Section 3.3. For each couple of modal numbers $(n, l)$, only one value is reported, relevant to the nonvanishing frequency shift. Results in Table 4 show that the linearized model is suitable for accurately evaluating frequency shifts of an imperfect rotor when imperfections are sufficiently small; for $\gamma \leq 0.1 \%$ the relative errors range from $0.3 \%$ to $2.5 \%$, increasing with mode number $n$. The relative difference between frequency shifts becomes larger for larger values of $\gamma$ together with higher values of mode number $n$; for an increased accuracy a refined theory may be used, which can be derived by retaining also the higher-order terms in the perturbation expansion performed in Section 3.2.
Accordingly, a nonlinear theory would be obtained, whose solution would require a numerical iterative procedure.

4.5. Eigenmode Analysis. In this section the eigenmodes of the rotor described in Section 4.1, made imperfect by increasing the linear mass density of its 4 th blade by $\gamma=$ $1 \%$, are evaluated. For the sake of comparison, both the HOM model and the L-HOM model are employed in the analysis, and their results are compared with the eigenmodes computed by using the finite-element formulation of the EB model, used as a benchmark. In Figure 4 the eigenmodes relevant to mode numbers $n=2 \cdots 5$ and $l=1$ are reported; in particular, for each couple of modal number $(n, l)$ only the eigenmode corresponding to a nonvanishing frequency shift $\delta \omega_{n, l}$ exhibits a vibration localization and is reported in the figure. All the modal shapes have been normalized using the same procedure: in particular, the modulus of the complex Fourier coefficient relevant to the dominant mode (i.e., the $n$th +1 Fourier coefficient for a eigenmode of mode number $n$ ) has been set to 1 . Finally, the eigenmodes have been all rescaled by a factor of 0.2 to be clearly shown in the figure.

The homogenized model turns out to be suitable for studying the localization phenomenon in imperfect rotors: in fact, as shown in Figure 4, the localization effect due to the imperfection placed on the fourth blade clearly appears, increasing with the increase of the modal number $n$. It turns out that the HOM model and the L-HOM model are both in good agreement with the reference EB model, with the LHOM model slightly underestimating the modal amplitude around the imperfect blade with respect to the HOM model.

\section{Conclusions}

In this paper a homogenized model has been proposed, suitable for the dynamical analysis of mistuned bladed rotors. The model seems to be a good compromise between accuracy and simplicity; indeed, it considers the distributed elasticity 
TABLE 4: Relative error between frequency shifts relevant to the imperfect rotor, evaluated according to the HOM model and the L-HOM model. The imperfection is introduced by increasing the mass of the fourth blade of the perfect rotor described in Section 4.1 by $\gamma=0.05 \%$, $0.1 \%, 0.5 \%$, and $1 \%$, with respect to its nominal value. Modal frequencies relevant to modal numbers $n=2 \cdots 5$ and $l=1$.

\begin{tabular}{lcccc}
\hline & $n=2$ & $n=3$ & $n=4$ & $n=5$ \\
\hline$\gamma=0.05 \%$ & 0.0032 & 0.0054 & 0.0087 & 0.0125 \\
$\gamma=0.1 \%$ & 0.0064 & 0.0108 & 0.0173 & 0.0250 \\
$\gamma=0.5 \%$ & 0.0319 & 0.0539 & 0.0860 & 0.1233 \\
$\gamma=1 \%$ & 0.0665 & 0.1122 & 0.1777 & 0.2507 \\
\hline
\end{tabular}

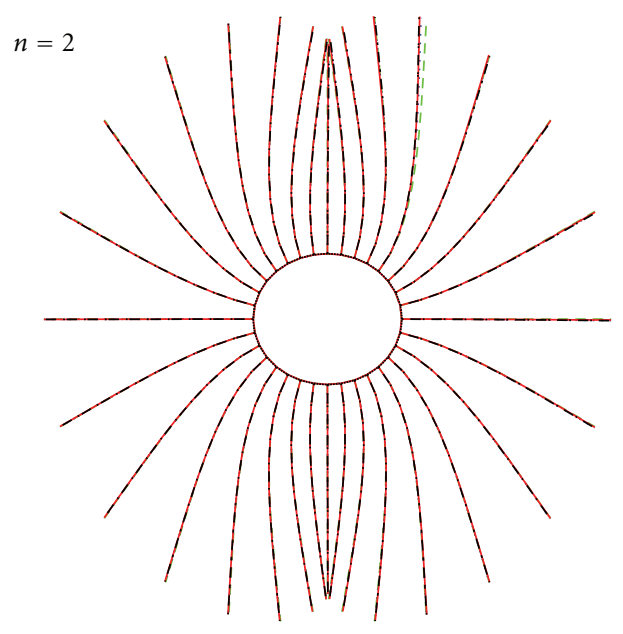

(a)

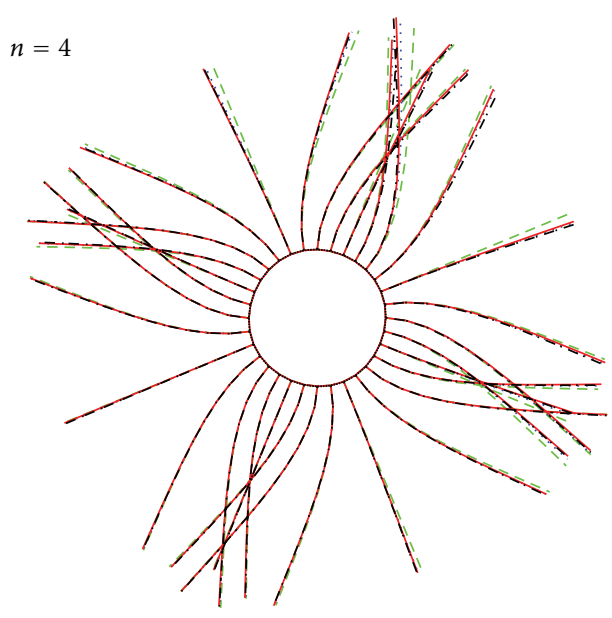

(c)

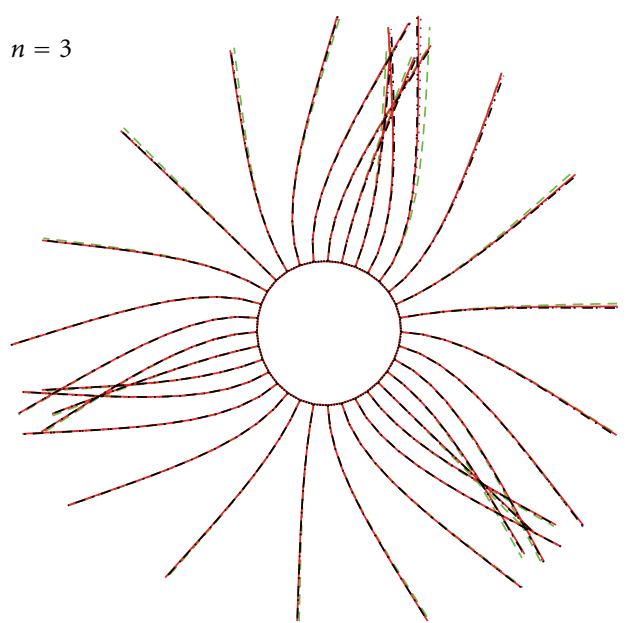

(b)

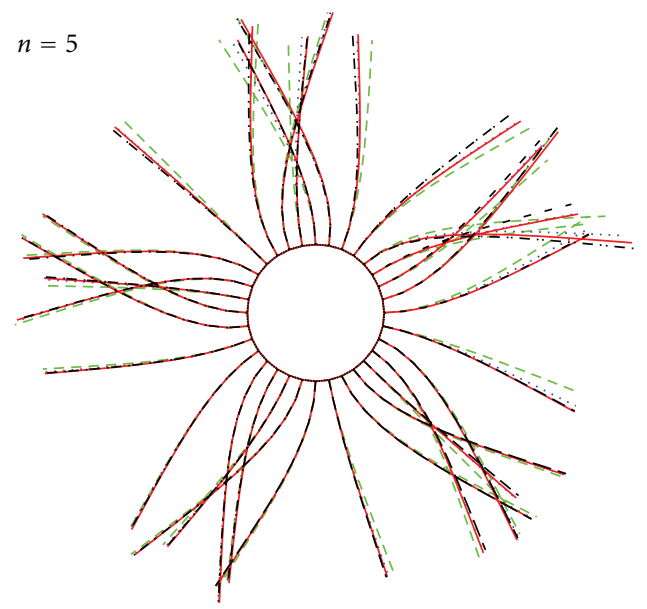

(d)

FIgURE 4: Eigenmodes of the imperfect rotor, evaluated according to the HOM model, the L-HOM model, and the EB model. The imperfection is introduced by increasing the mass of the fourth blade of the perfect rotor described in Section 4.1 by $\gamma=1 \%$, with respect to its nominal value. - - green: perfect rotor, — red: imperfect rotor using HOM model, - - blue: imperfect rotor using L-HOM model, - · black: imperfect rotor using the EB model.

of the blades and the coupling between a single blade and the other ones, due to the presence of a flexible support disk. On the other end, it is a continuous model, and thus it is computationally more effective than classical finite element models. Numerical simulations have been reported, showing the capability of the model in evaluating the frequency split and vibration localization phenomenon arising in the presence of mistuning. The proposed model may be an effective tool for the design and the parametric analysis of bladed rotors.

\section{References}

[1] D. L. Thomas, "Dynamics of rotationally periodic structures," International Journal for Numerical Methods in Engineering, vol. 14, no. 1, pp. 81-102, 1979.

[2] I. Y. Shen, "Vibration of rotationally periodic structures," Journal of Sound and Vibration, vol. 172, no. 4, pp. 459-470, 1994.

[3] A. J. Fricker and S. Potter, "Transient forced vibration of rotationally periodic structure," International Journal for Numerical Methods in Engineering, vol. 17, no. 7, pp. 957-974, 1981. 
[4] C. W. Cai, Y. K. Cheung, and H. C. Chan, "Uncoupling of dynamic equations for periodic structures," Journal of Sound and Vibration, vol. 139, no. 2, pp. 253-263, 1990.

[5] D. J. Ewins, "The effects of detuning upon the forced vibrations of bladed disks," Journal of Sound and Vibration, vol. 9, no. 1 , pp. 65-79, 1969.

[6] A. Sinha, "Calculating the statistics of forced response of a mistuned bladed disk assembly," AIAA Journal, vol. 24, no. 11, pp. 1797-1801, 1986.

[7] C. Pierre and E. H. Dowell, "Localization of vibrations by structural irregularity," Journal of Sound and Vibration, vol. 114, no. 3, pp. 549-564, 1987.

[8] B. W. Huang, "Effect of number of blades and distribution of cracks on vibration localization in a cracked pre-twisted blade system," International Journal of Mechanical Sciences, vol. 48, no. 1, pp. 1-10, 2006.

[9] Y. J. Chiu and S. C. Huang, "The influence on coupling vibration of a rotor system due to a mistuned blade length," International Journal of Mechanical Sciences, vol. 49, no. 4, pp. 522532, 2007.

[10] E. P. Petrov and D. J. Ewins, "Analysis of the worst mistuning patterns in bladed disk assemblies," Journal of Turbomachinery, vol. 125, no. 4, pp. 623-631, 2003.

[11] M. P. Mignolet, W. Hu, and I. Jadic, "On the forced response of harmonically and partially mistuned bladed disks. Part I: harmonic mistuning," International Journal of Rotating Machinery, vol. 6, no. 1, pp. 29-41, 2000.

[12] M. P. Mignolet, W. Hu, and I. Jadic, "On the forced response of harmonically and partially mistuned bladed disks. Part II: partial mistuning and applications," International Journal of Rotating Machinery, vol. 6, no. 1, pp. 43-56, 2000.

[13] J. Tang and K. W. Wang, "Vibration delocalization of nearly periodic structures using coupled piezoelectric networks," Journal of Vibration and Acoustics, vol. 125, no. 1, pp. 95-108, 2003.

[14] H. H. Yoo, J. Y. Kim, and D. J. Inman, "Vibration localization of simplified mistuned cyclic structures undertaking external harmonic force," Journal of Sound and Vibration, vol. 261, no. 5, pp. 859-870, 2003.

[15] X. Fang, J. Tang, E. Jordan, and K. D. Murphy, "Crack induced vibration localization in simplified bladed-disk structures," Journal of Sound and Vibration, vol. 291, no. 1-2, pp. 395-418, 2006.

[16] Y. J. Chan and D. J. Ewins, "Management of the variability of vibration response levels in mistuned bladed discs using robust design concepts. Part 1: parameter design," Mechanical Systems and Signal Processing, vol. 24, no. 8, pp. 2777-2791, 2010.

[17] L. F. Wagner and J. H. Griffin, "A continuous analog model for grouped-blade vibration," Journal of Sound and Vibration, vol. 165, no. 3, pp. 421-438, 1993.

[18] P. Bisegna and G. Caruso, "Dynamical behavior of disordered rotationally periodic structures: a homogenization approach," Journal of Sound and Vibration, vol. 330, no. 11, pp. 26082627, 2011.

[19] P. Bisegna and G. Caruso, "Optimization of a passive vibration control scheme acting on a bladed rotor using an homogenized model," Structural and Multidisciplinary Optimization, vol. 39, no. 6, pp. 625-636, 2009. 

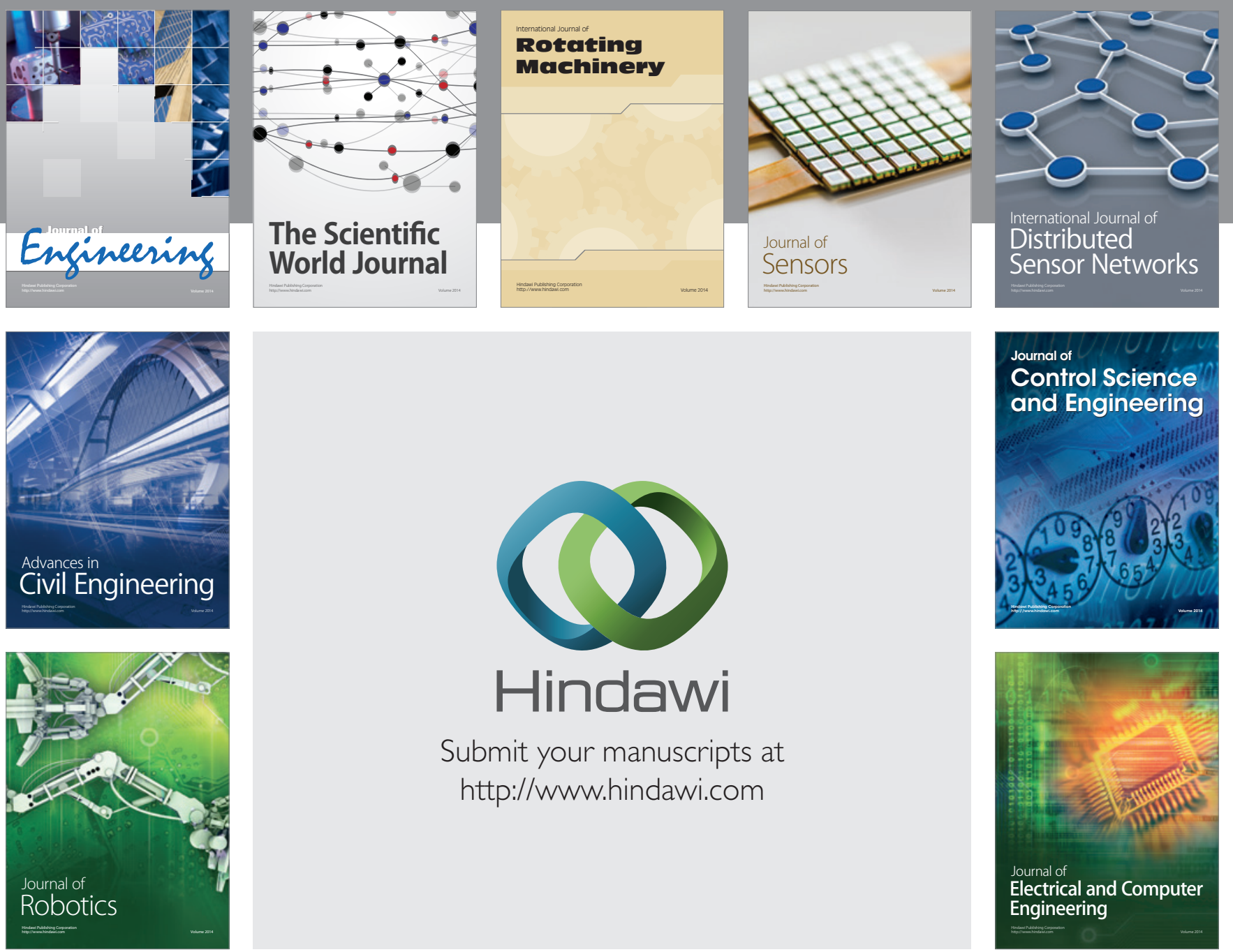

Submit your manuscripts at

http://www.hindawi.com
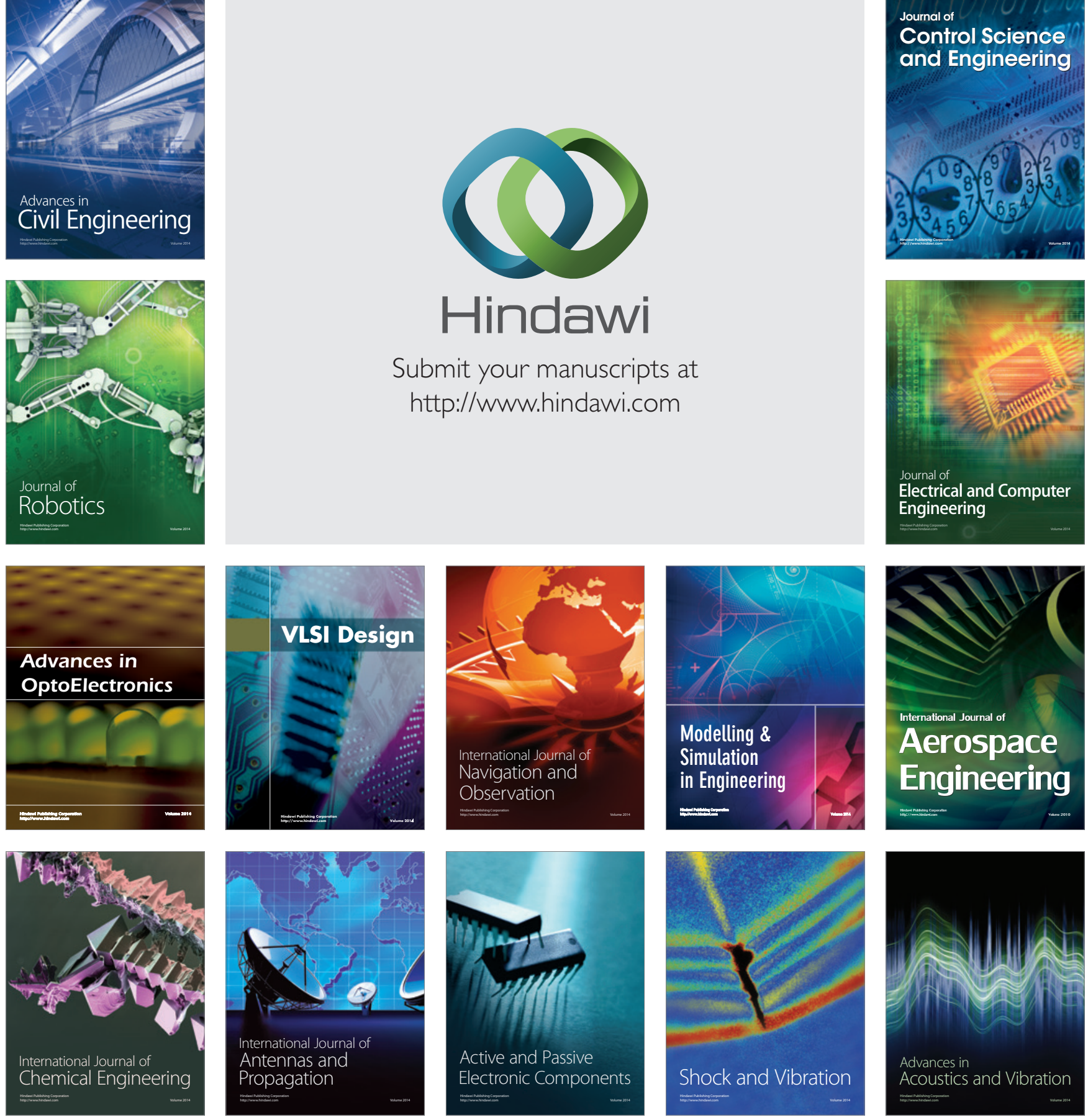